Bangladesh J. Zool. 41(2): 113-133, 2013

\title{
ICHTHYOFAUNA OF THE RIVER HALDA, CHITTAGONG, BANGLADESH
}

\author{
Mohammad Ali Azadi ${ }^{*}$ and Mohammad Arshad-Ul-Alam¹ \\ Department of Zoology, University of Chittagong, Chittagong-4331, Bangladesh
}

\begin{abstract}
Eighty-three species of finfish belonging to 13 Orders, 35 Families, and 69 genera including three exotic species, and 10 species of shellfish (crustaceans) under one order, 3 families and 3 genera were identified from the River Halda during September 2004 to December 2011. Thus a total of 93 species of Ichthyofauna (finfish and shellfish) were recorded from this river during the seven years (2004-2011) of investigation. Maximum number of finfish species were recorded under the family Cyprinidae (19 species) followed by the family Gobiidae (11 species), whereas maximum number of shellfish species were recorded under the family Palaemonidae ( 8 species) followed by Penaeidae $(1$ species $)$ and Portunidae (1 species). Of the 83 finfish species, three belonged to the critically endangered, nine to the endangered and eight to the vulnerable category according IUCN (2000). Four species of finfish were new records from inland waters of Bangladesh. A systematic list of the ichthyofauna with local and English names, and habitat preference are provided.
\end{abstract}

Key words: Ichthyofauna, Halda River, fin fishes, shellfishes

\section{INTRODUCTION}

The tidal River Halda $\left(22^{\circ} 54^{\prime} \mathrm{N}\right.$ and $91^{04} 8^{\prime} \mathrm{E}$ to $22^{\circ} 24^{\prime} \mathrm{N}$ and $\left.91^{\circ} 53^{\prime} \mathrm{E}\right)$ is one of the tributaries of the River Karnaphuli and well known as the country's main spawning ground of major carps from which naturally fertilized eggs of carps are collected and hatched in mud-made scoops on the river bank for aquaculture purposes (Patra and Azadi 1985b, Khan and Azadi 2006). Originated from the Khagrachari hills and after traversing $88 \mathrm{~km}$, the river opens into the River Karnaphuli about half kilometer north of the Kalurghat Bridge on the River Karnaphuli. Due to anthropogenic and natural causes the spawn fishery (fry production) of major carps of Halda River has recently declined tremendously, $2470 \mathrm{~kg}$ in 1945 to $625 \mathrm{~kg}$ in 2013 (Khan and Azadi 2006, DoF 2013). Description of the other features of the River Halda is given elsewhere (Azadi and Arshad-ul-Alam 2011). The inland fisheries of Bangladesh have a diverse fish fauna of 264 species (Rahman 2005) which is the third richest in the world after China and India (Talwar and Jhingran 1991). The vast inland water of 4.57 million hectare with 3.86 million hectare floodplain-rivers-estuarine system provides an excellent ecologically diverse habitat for this taxonomically varied fish fauna. River Halda is a species rich habitat, isolated from GangesBrahmaputra river systems with physical barrier. A heterogeneous assemblage of floodplain, riverine, estuarine and marine fishes is found in this river with

*Corresponding author: <maazadi@yahoo.com>. ${ }^{1}$ Bandarban Government College, Bandarban Hill District, Bangladesh 
strong dominance of a few species. Some research were conducted on different ecological and fisheries aspects of the River Halda, such as, egg collection (Ahmed 1948, 1955, Patra and Azadi 1984), limnology (Patra and Azadi 1985a), hydrological factors (Patra and Azadi 1985b), ecological studies on plankton (Patra and Azadi 1987), management of spawn fishery (Azadi 2004), revival of carp natural spawning ground (Khan and Azadi 2006) biodiversity and field sites of fish (Azadi and Arshad-ul-Alam 2011), Fishing intensity (Arshadul Alam 2013). Under the Bangladesh Government's Irrigation and Fishery Development Projects some short term works, such as, different fisheries aspects of Halda and Ichamati Rivers and its adjacent flood plains combined were done by Ali and Morris (1977, 1978), on catch survey, fish and prawn fauna by Rainboth (1978), and fishing gears by Chong (1979). Besides, a few working documents of Irrigation and flood contrl Project, there is no other information on the fish and fishery of Halda River before or after the major interventions (12 sluice gates which blocked 12 major tributaries at lower Halda and $47 \mathrm{~km}$ embankment during 1975-76 and 1982-1983) in the river Halda.

Works on the freshwater fish fauna of Bangladesh are represented by the works of Ahmed (1953), Bhuiyan (1964), Doha (1973), Ahmed and Hasan (1981), Shafi and Quddus (1982), Haroon et al. (1989), Hafizuddin et al. (1989), Rahman (1975, 1989, 2005) and Hossain (2013).

The present investigation was carried out to know the ichthyofauna (finfish and shellfish species) and their (IUCN 2000) status (endangered, critically endangered, vulnerable) in the river Halda and the findings might be useful to the researchers, planners and biologists as the river is declared fish sanctuary in 2007.

\section{MATERIAL AND METHODS}

For the study of finfish and shellfish species of the Halda River a total of 1217 samples was collected fortnightly during September 2004 to December 2011 directly from the fishermen catch caught by 34 types of different fishing gears. The sampling area covered all the fishing zones (39 kilometers) from Nazirhat Bridge to Halda mouth opening in the Karnaphuli river near Mohra point. Photographs of these catches were taken in fresh condition in the field. External features with colour patterns were noted in fresh condition. Specimens were preserved in $7 \%$ formaldehyde solution and kept in the Department of Zoology, University of Chittagong for further study and future reference.

The classificatory scheme of fish has been followed after Berg (1940) with a few modifications by Lagler (1962) and Rahman (2005). Identification and classification of fin fish were done with the help of Day (1889), Munro (1955), 
Bhuiyan (1964), Jayaram (1981), Shafi and Quddus (1982) and Rahman (2005) while for crustaceans help was taken from Ahmed (1957), George (1969), Howlader (1976), Ficnham and Wickins (1976) and Shafi and Quddus (1982). Zoological names of all the genera and species were listed in accordance with the rules and principles as set forth in the international code. Vernacular names are also provided. Website of fishbase was used for information on synonyms. The threat status of the fish species mentioned in the result was as per IUCN Bangladesh (2000). Systematic list with local name, common English name and habitat preference were provided. National biodiversity status (categories of threat) as per IUCN was also cited for fish species.

\section{RESULTS AND DISCUSSION}

During seven years of study period (September 2004 to December 2011) a total of 83 finfish species under 13 orders and 35 families and a total of 10 shellfish (9 prawns and one crab) under 1 order and 3 families (Plates 1 to 5) were identified from the River Halda. The systematic index is presented here.

\section{Phylum: Chordata}

\section{Subphylum: Vertebrata}

Class: Osteichthyes

\section{Order: 1. Anguilliformes}

\section{Familly: 1. Anguillidae}

1. Anguilla bengalensis (Gray, 1831) (Plate 1, Sl. No. 1)

Local name: Bara Baim, Cheloch; English name: Indian Long-fin Eel; Habitat: River, estuary and sea (Migratory catadromous); IUCN status: Vulnerable.

\section{Familly: 2. Moringuidae}

2. Moringua raitaborua (Hamilton, 1822) (Plate 1, Sl. No. 2)

Local name: Rata Boura, Baim; English name: Purple Spaghetti Eel; Habitat: River and estuary.

3. Moringua macrocephalus (Bleeker, 1863) (Plate 1, No. 3)

Local name: Baim; English name: Spaghetti Eel; Habitat: Estuary and sea. Not reported by Shafi and Quddus (1982), IUCN (2000) and Rahman (2005).

\section{Familly: 3. Ophichthidae}

4. Pisodonophis boro (Hamilton, 1822) (Plate 1, No. 4)

Local name: Nol Baim, Hijara; English name: Rice-paddy Eel; Habitat: Sea, estuary and river. 


\section{Order: 2. Clupeiformes}

\section{Familly: 4. Clupeidae}

5. Corica soborna Hamilton, 1822 (Plate 1, No. 5)

Local name: Kachki; English name: Ganges River Sprat; Habitat: River, Kaptai lake.

6. Gudusia chapra (Hamilton, 1822) (Plate 1, No. 6)

Local name: Chapila; English name: Indian River Shad; Habitat: Wide freshwater.

7. Tenualosa ilisha (Hamilton, 1822) (Plate 1, No. 7)

Local name: Illish; English name: Hilsa Shad; Habitat: Sea, estuary and river.

Familly: 5. Engraulidae

8. Setipinna phasa (Hamilton, 1822) (Plate 1, No. 8)

Local name: Phaissha; English name: Gangetic Hairfin Anchovy; Habitat: River, Estuary, Kaptai lake.

\section{Order: 3. Osteoglossiformes}

\section{Familly: 6. Notopteridae}

9. Notopterus notopterus (Pallas, 1769) (Plate 1, No. 9)

Local name: Foli; English name: Bronze Feather back; Habitat: Flood plain pond, lake and beel; IUCN status: Vulnerable.

\section{Order: 4. Cypriniformes}

\section{Familly: 7. Cyprinidae}

10. Labeo ariza (Hamilton, 1807) (Plate 1, No. 10)

Local name: Lasso, Bata, Raik; English name: Ariza Labeo; Habitat: Wide freshwater. Not reported by Shafi and Quddus (1982), IUCN (2000) and Rahman (2005).

11. Labeo calbasu (Hamilton, 1822) (Plate 1, No. 11)

Local name: Kalibaush; English name: Black Rohu; Habitat: River; IUCN status: Endangered.

12. Labeo gonius (Hamilton, 1822) (Plate 1, No. 12)

Local name: Shada Ghoni; English name: Kuria Labeo; Habitat: River; IUCN status: Endangered.

13. Labeo rohita (Hamilton, 1822) (Plate 1, No. 13)

Local name: Rui; English name: Ruho; Habitat: River.

14. Catla catla (Hamilton, 1822) (Plate 1, No. 14)

Local name: Catla; English name: Catla; Habitat: River. 


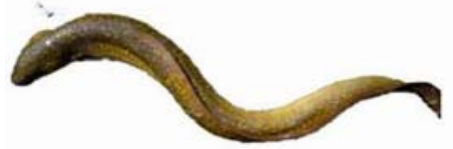

1. Anguilla bengalensis

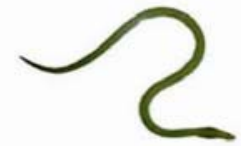

4. Pisodonophis boro

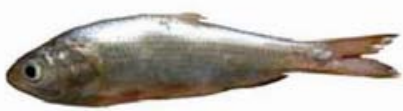

4. Tenualosa ilisha

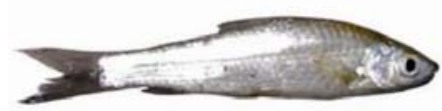

10. Labeo ariza

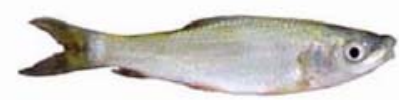

13. Labeo rohita

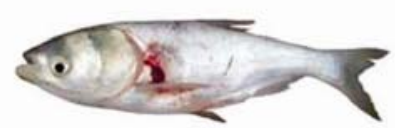

16. Aristichthys nobilis

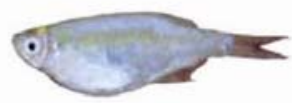

19. Chela laubuca

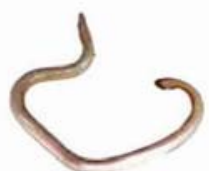

2. Moringua raitaboura

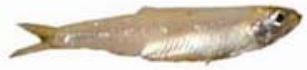

5. Corica soborna

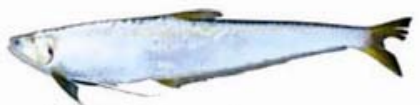

8. Setipinna phasa

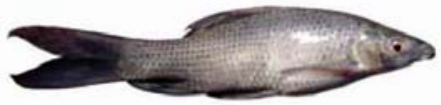

11. Labeo calbasu

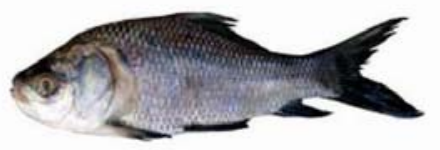

14. Catla catla

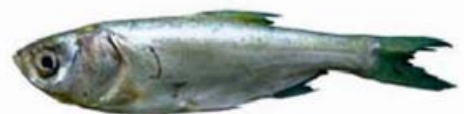

17. Hypophthalmichthys molitrix

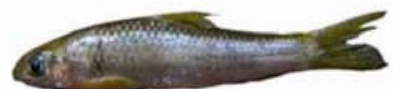

20. Crossocheilus latius

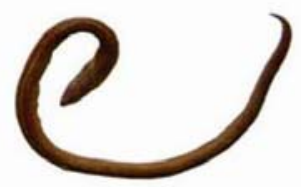

3. Moringua macrocephalus

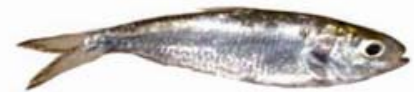

6. Gudusia chapra

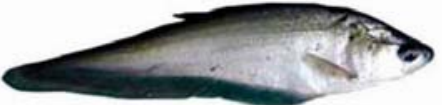

9. Notopterous notopterous

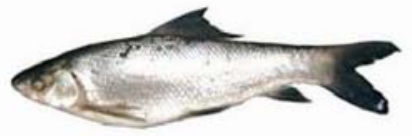

12. Labeo gonius

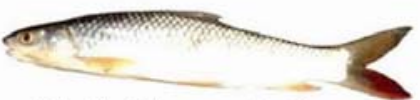

15. Cirrhinus mrigala

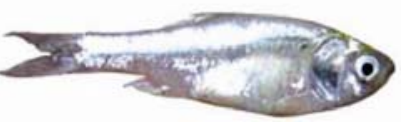

18. Amblypharyngodon mola

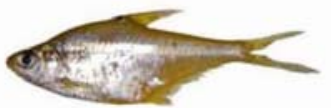

21. Osteobrama cotio

Plate 1. Fin fishes of the River Halda

15. Cirrhinus mrigala (Hamilton, 1822) (Plate 1, No. 15) Local name: Mrigal; English name: Mrigal; Habitat: River.

16. Aristichthys nobilis (Richardson, 1845) (Plate 1, No. 16) Local name: Bighead; English name: Bighead Carp; Habitat: River (exotic).

17. Hypophthalmichthys molitrix (Valenciennes, 1844) (Plate 1, No. 17) Local name: Silver Carp; English name: Silver Carp; Habitat: River (exotic). 
18. Amblypharyngodon mola (Hamilton, 1822) (Plate 1, No. 18) Local name: Mola; English name: Mola Carplet; Habitat: Wide freshwater.

19. Chela laubuca (Hamilton, 1822) (Plate 1, No. 19) Local name: Chela; English name: Indian Glass Barb; Habitat: Wide freshwater; IUCN status: Endangered.

20. Crossocheilus latius (Hamilton, 1822) (Plate 1, No. 20)

Local name: Sajani, Kala Bata; English name: Latia; Habitat: Wide freshwater; IUCN status: Endangered.

21. Osteobrama cotio (Hamilton, 1822) (Plate 1, No. 21)

Local name: Nak-kata; English name: Cotio; Habitat: Wide freshwater; IUCN status: Endangered.

22. Puntius sophore (Hamilton, 1822) (Plate 2, No. 22)

Local name: Jathi Punti; English name: Spotfin Swamp Barb; Habitat: Wide freshwater.

23. Puntius ticto (Hamilton, 1822) (Plate 2, No. 23)

Local name: Tit Punti; English name: Ticto Barb; Habitat: Wide freshwater; IUCN status: Vulnerable.

24. Puntius gelius (Hamilton, 1822) (Plate 2, No. 24)

Local name: Geli Punti; English name: Golden Dwarf Barb; Habitat: Wide freshwater.

25. Puntius conchonius (Hamilton, 1822) (Plate 2, No. 25)

Local name: Kanchon Punti, Tit Punti; English name: Rosy Barb; Habitat: Wide freshwater.

26. Rasbora rasbora (Hamilton, 1822) (Plate 2, No. 26)

Local name: Darkina; English name: Gangetic Scissortail Rasbora; Habitat: Wide freshwater; IUCN status: Endangered.

27. Salmostoma bacaila (Hamilton, 1822) (Plate 2, No. 27)

Local name: Chela, Bachkuitta; English name: Large Razorbelly Minnow; Habitat: Wide freshwater.

28. Salmostoma phulo (Hamilton, 1822) (Plate 2, No. 28)

Local name: Chela, Bachkuitta; English name: Finescale Razorbelly Minnow; Habitat: Wide freshwater.

Familly: 8. Cobitidae

29. Lepidocephalichthys guntea (Hamilton, 1822) (Plate 2, No. 29)

Local name: Gutum, Puiya; English name: Guntea Loach; Habitat: Wide freshwater. 


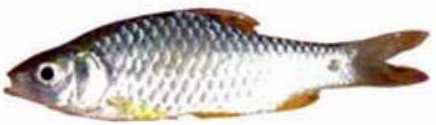

22. Puntius sophore

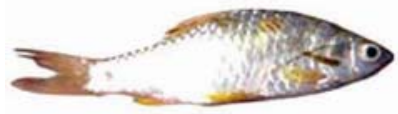

25. Puntius conchonius

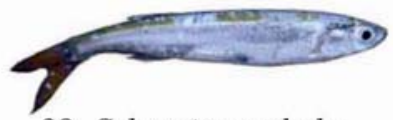

28. Solmostoma phulo

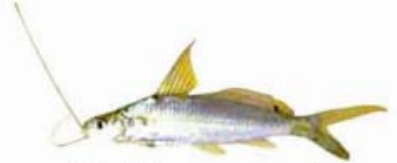

31. Mystus cavasius

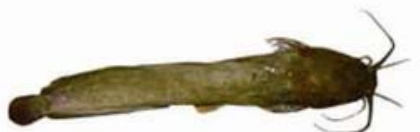

34. Clarias batrachus

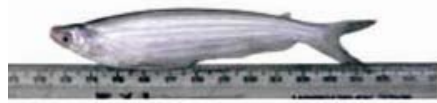

37. Ailia coila

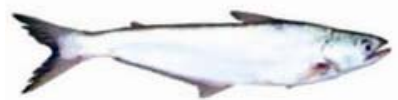

40. Eutropiichthys vacha

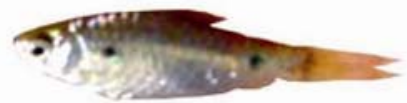

23. Puntius ticto

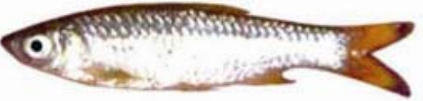

26. Rasbora rasbora

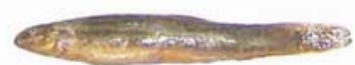

29. Lepidocephalichthys guntea

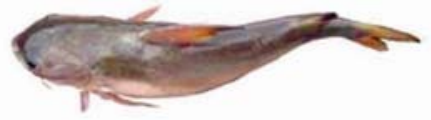

32. Mystus gulio

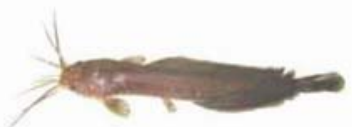

38. Heteropneustes fossilis

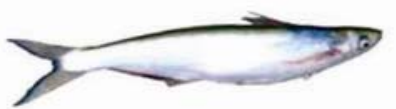

38. Clupisoma garua

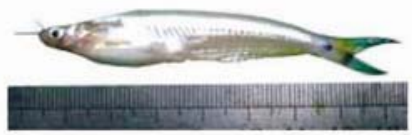

41. Neotropius atherinoides

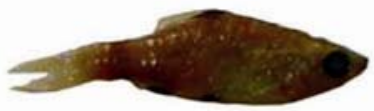

24. Puntius gelius

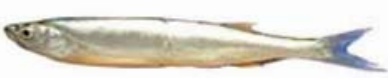

27. Salmostoma bacaila

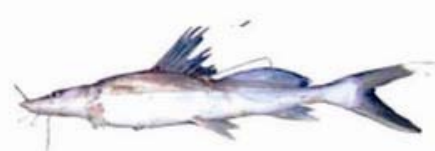

30. Sperata aor

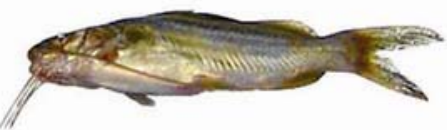

33. Mystus vittatus

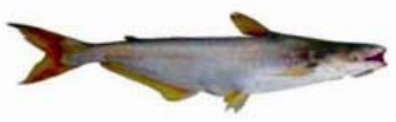

36. Pangasius pangasius

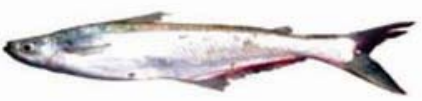

39. Eutropiichthys murius

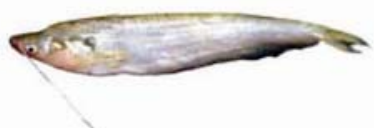

42. Ompok pabda

Plate 2. Fin fishes of the River Halda (continued)

\section{Order: 5. Siluriformes}

\section{Familly: 9. Bagridae}

30. Sperata aor (Hamilton, 1822) (Plate 2, No. 30)

Local name: Ayre; English name: Long-whiskered Catfish; Habitat: Wide freshwater; IUCN status: Vulnerable.

31. Mystus cavasius (Hamilton, 1822) (Plate 2, No. 31) 
Local name: Gulsha, Guilla; English name: Gangetic Mystus; Habitat: Wide freshwater; IUCN status: Vulnerable.

32. Mystus gulio (Hamilton, 1822) (Plate 2, No. 32)

Local name: Nuna Tengra, Shada Tengra; English name: Longwhiskered Catfish; Habitat: Sea, estuary and river (Anadromous migratory).

33. Mystus vittatus (Bloch, 1794) (Plate 2, No. 33)

Local name: Tengra; English name: Striped Dwarf Catfish; Habitat: Wide freshwater.

\section{Familly: 10. Clariidae}

34. Clarias batrachus (Linnaeus, 1758) (Plate 2, No. 34)

Local name: Magur; English name: Walking Catfish; Habitat: Flood plain and beel.

\section{Familly: 11. Heteropneustidae}

35. Heteropneustes fossilis (Bloch, 1794) (Plate 2, No. 35)

Local name: Shingi; English name: Stinging Catfish; Habitat: Flood plain and beel.

\section{Familly: 12. Pangasiidae}

36. Pangasius pangasius (Hamilton, 1822) (Plate 2, No. 36)

Local name: Pangas; English name: Yellowtail Catfish; Habitat: River and estuary; IUCN status: Critically endangered.

\section{Familly: 13. Schilbeidae}

37. Ailia coila (Hamilton, 1822) (Plate 2, No. 37)

Local name: Banspata, Kajuli; English name: Gangetic Ailia; Habitat: River, Kaptai lake.

38. Clupisoma garua (Hamilton, 1822) (Plate 2, No. 38)

Local name: Gharua; English name: Garua Bachcha; Habitat: River ; IUCN status: Critically endangered.

39. Eutropiichthys murius (Hamilton, 1822) (Plate 2, No. 39)

Local name: Muri Bacha; English name: Murius Vacha; Habitat: River.

40. Eutropiichthys vacha (Hamilton, 1822) (Plate 2, No. 40)

Local name: Bacha; English name: Batchwa Vacha; Habitat: River, Kaptai lakre; IUCN status: Critically endangered.

41. Neotropius atherinoides (Bloch, 1794) (Plate 2, No. 41) 
Local name: Tinkata Batasi, Hil Guilla; English name: Indian Potasi; Habitat: River.

\section{Familly: 14. Siluridae}

42. Ompok pabda (Hamilton, 1822) (Plate 2, No. 42)

Local name: Pabda; English name: Pabdah Catfish; Habitat: Wide freshwater; IUCN status: Endangered.

43. Wallago attu (Bloch \& Schneider, 1801) (Plate 3, No. 43)

Local name: Boal; English name: Wallago; Habitat: Wide freshwater.

\section{Order: 6. Beloniformes}

Familly: 15. Adrianichthyidae

44. Oryzias dancena (Hamilton, 1822) (Plate 3, No. 44)

Local name: Chela, Kagoji gura; English name: Estuarine Ricefish; Habitat: Wide freshwater. Not reported by Shafi and Quddus (1982), IUCN (2000) and Rahman (2005).

\section{Familly: 16. Hemiramphidae}

45. Hyporhamphus limbatus (Valenciennes, 1847) (Plate 3, No. 45)

Local name: Thuitta; English name: Congaturi Halfbeak; Habitat: Wide freshwater.

\section{Familly: 17. Belonidae}

46. Xenentodon cancila (Hamilton, 1822) (Plate 3, No. 46)

Local name: Kakila; English name: Freshwater Garfish; Habitat: Wide freshwater.

\section{Order: 7. Cyprinodontiformes}

\section{Familly: 18. Aplocheilidae}

47. Aplocheilus panchax (Hamilton, 1822) (Plate 3, No. 47)

Local name: Chukkuni; English name: Blue Panchax; Habitat: Flood plain and beel.

\section{Order: 8. Syngnathiformes}

\section{Familly: 19. Syngnathidae}

48. Microphis cuncalus (Hamilton, 1822) (Plate 3, No. 48)

Local name: Kumirer Khil; English name: Crocodile-tooth Pipefish; Habitat: River and Estuary.

\section{Order: 9. Perciformes}

Familly: 20. Ambassidae

49. Chanda nama Hamilton, 1822 (Plate 3, No. 49) 
Local name: Chanda; English name: Elongate Glass-perchlet; Habitat: Wide freshwater; IUCN status: Vulnerable.

50. Parambassis ranga (Hamilton, 1822) (Plate 3, No. 50)

Local name: Lal Chanda; English name: Indian Glassy Fish; Habitat: Wide freshwater; IUCN status: Vulnerable.

51. Pseudambassis baculis (Hamilton, 1822) (Plate 3, No. 51)

Local name: Chanda; English name: Himalayan Glassy Perchlet; Habitat: Wide freshwater.

\section{Familly: 21. Scatophagidae}

52. Scatophagus argus (Linnaeus, 1766) (Plate 3, No. 52)

Local name: Bishtara; English name: Spotted Scat; Habitat: Estuary and sea; IUCN status: Endangered.

\section{Familly: 22. Sciaenidae}

53. Johnius coitor (Hamilton, 1822) (Plate 3, No. 53)

Local name: Poa; English name: Coitor Croaker; Habitat: River, Estuary and Kaptai lake.

54. Macrospinosa cuja (Hamilton, 1822) (Plate 3, No. 54)

Local name: Poa; English name: Cuja Bola; Habitat: Sea, estuary and river (anadromous migratory).

\section{Familly: 23. Cichlidae}

55. Oreochromis niloticus (Linnaeus, 1758) (Plate 3, No. 55)

Local name: Nilotica; English name: Nile Tilapia; Habitat: Freshwater (exotic).

\section{Familly: 24. Anabantidae}

56. Anabas testudineus (Bloch, 1792) (Plate 3, No. 56)

Local name: Koi; English name: Climbing Perch; Habitat: Freshwater.

\section{Familly: 25. Sillaginidae}

57. Sillaginopsis panijus (Hamilton, 1822) (Plate 3, No. 57)

Local name: Sundra, Tular Dandi; English name: Gangetic Sillago;

Habitat: Estuary and sea.

\section{Familly: 26. Gobiidae}

58. Glossogobius giuris (Hamilton, 1822) (Plate 3, No. 58)

Local name: Byla; English name: Tank Goby; Habitat: Wide freshwater.

59. Brachygobius nunus (Hamilton, 1822) (Plate 3, No. 59)

Local name: Byla-gura, Nuna Byla; English name: Bumblebee Goby; Habitat: River and estuary. 


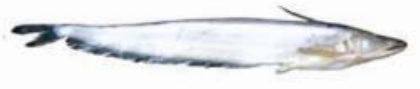

43. Wallago attu

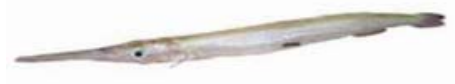

46. Xenentodon cancila

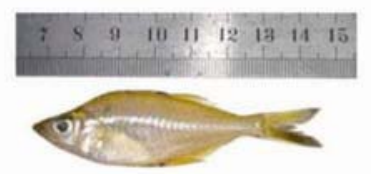

49. Chanda nama

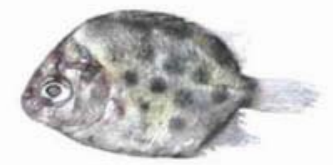

52. Scatophagus argus

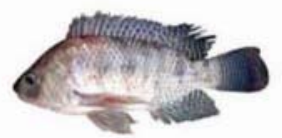

55. Oreochromis niloticus

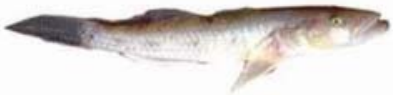

58. Glossogobius giuris

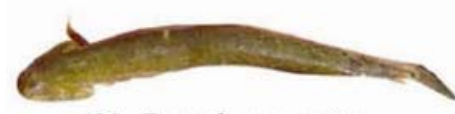

61. Pseudapocryptes elongatus

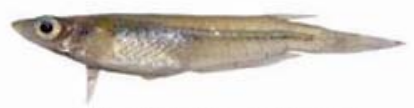

44. Oryzias dancena

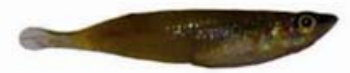

47. Aplocheilus panchax

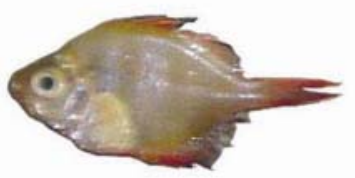

50. Parambassis ranga

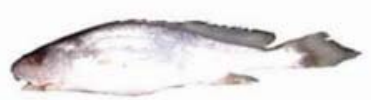

53. Johnius coitor

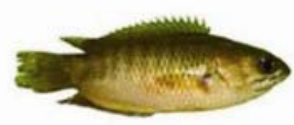

56. Anabas testudineus

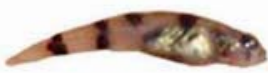

59. Brachygobius nunus

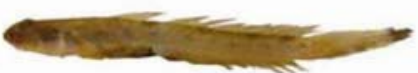

62. Parapocryptes serperaster

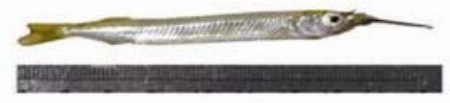

45. Hyporhamphus limbatus

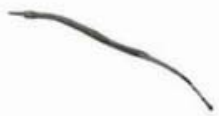

48. Microphis cuncalus

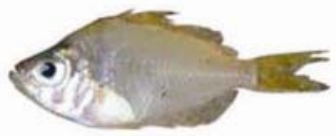

51. Pseudambassis baculis

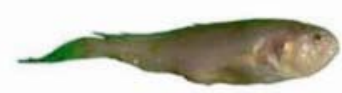

54. Macrospinosa cuja

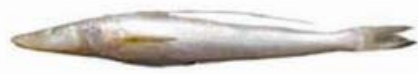

57. Sillaginopss panijus

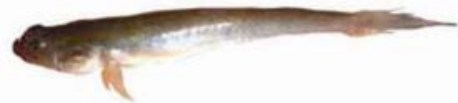

60. Apocryptes bato

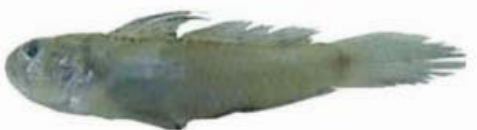

63. Oxyurichthys microlepis

Plate 3. Fin fishes of the River Halda (continued)

60. Apocryptes bato (Hamilton, 1822) (Plate 3, No. 60) Local name: Fhul Chiring; English name: Goby; Habitat: River and estuary.

61. Pseudapocryptes elongatus (Cuvier, 1816) (Plate 3, No. 61) Local name: Fhul Chiring; English name: Goby; Habitat: Estuary and sea. Not reported by Shafi and Quddus (1982) and IUCN (2000).

62. Parapocryptes serperaster (Richardson, 1846) (Plate 3, No. 62) 
Local name: Dora Chiring; English name: Goby; Habitat: Estuary and sea.

63. Oxyurichthys microlepis (Bleeker, 1849) (Plate 3, No. 63)

Local name: Sabuj Chiring; English name: Maned Goby; Habitat: River and estuary.

64. Taenioides cirratus (Blyth, 1860) (Plate 4, No. 64)

Local name: Lal Chew; English name: Bearded Worm Goby; Habitat: River and estuary.

65. Odontamblyopus rubicundus (Hamilton, 1822) (Plate 4, No. 65)

Local name: Shada Chew; English name: Rubicundus Eel Goby; Habitat: River and estuary.

66. Ophieleotris aporos (Bleeker, 1854) (Plate 4, No. 66)

Local name: Burguni; English name: Aporos Sleeper, Snakehead Gudgeon; Habitat: River and estuary. Not reported by Shafi and Quddus (1982), IUCN (2000) and Rahman (2005).

67. Stigmatogobius sadanundio (Hamilton, 1822) (Plate 4, No. 67)

Local name: Burguni; English name: Gudgeon; Habitat: River and estuary.

68. Periophthalmodon schlosseri (Pallas, 1770) (Plate 4, No. 68)

Local name: Dok Mach, Dok Chiring; English name: Giant Mudskipper; Habitat: Estuary.

\section{Familly: 27. Osphronemidae}

69. Colisa fasciatus (Bloch \& Schneider, 1801) (Plate 4, No. 69)

Local name: Khalisha; English name: Banded Gourami; Habitat: Wide freshwater.

70. Trichogaster chuna (Hamilton, 1822) (Plate 4, No. 70)

Local name: Choto Khalisha; English name: Honey Gourami; Habitat: Wide freshwater.

\section{Familly: 28. Polynemidae}

71. Polynemus paradiseus Linnaeus, 1758 (Plate 4, No. 71)

Local name: Thopshe; English name: Paradise Threadfin; Habitat: Estuary.

\section{Familly: 29. Channidae}

72. Channa orientalis Bloch \& Schneider 1801 (Plate 4, No. 72) Local name: Cheng; English name: Walking Snakehead; Habitat: Flood plain and beel; IUCN status: Vulnerable. 


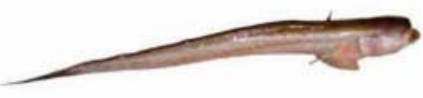

64. Taenioides cirratus

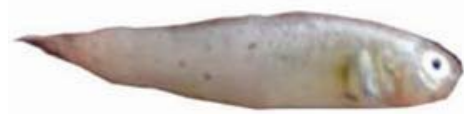

67. Stigmatogobius sadanundio

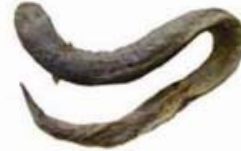

65. Odontamblyopus rubicundus

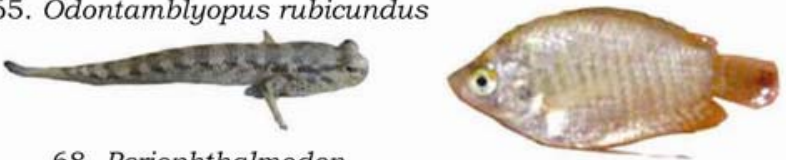

68. Periophthalmodon schlosseri

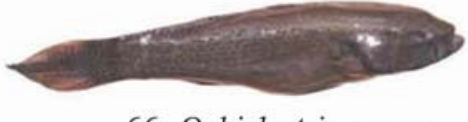

66. Ophieleotris aporos

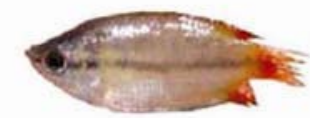

70. Trichogaster chuna

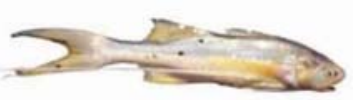

71. Polynemus paradiseus

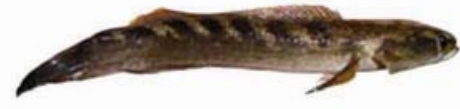

72. Channa orientalis

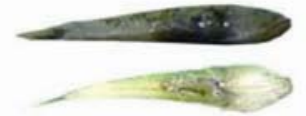

75. Opistognathus nigromarginatus

74. Channa striatus

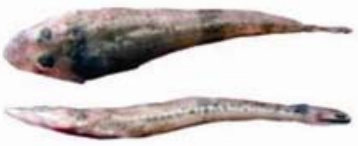

78. Platycephalus indicus

76. Sicamugil cascasia

77. Rhinomugil corsula

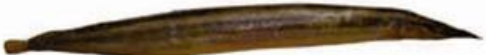

81. Macrognathus aculentus

80. Macrognathus pancalus

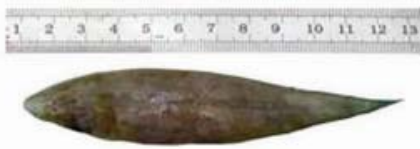

83. Cynoglossus cynoglossus

Plate 4. Fin fishes of the River Halda (continued)

73. Channa punctatus (Bloch, 1793) (Plate 4, No. 73)

Local name: Taki; English name: Spotted Snakehead; Habitat: Flood plain and beel.

74. Channa striatus (Bloch, 1793) (Plate 4, No. 74)

Local name: Shol; English name: Snakehead Murrel; Habitat: Flood plain and beel.

\section{Familly: 30. Opistognathidae}

75. Opistognathus nigromarginatus Rüppell, 1830 (Plate 4, No. 75) 
Local name: Bang Mach; English name: Birdled Jawfish; Habitat: Sea. Not reported by Shafi and Quddus (1982), IUCN (2000) and Rahman (2005).

\section{Order: 10. Mugiliformes}

\section{Familly: 31. Mugilidae}

76. Sicamugil cascasia (Hamilton, 1822) (Plate 4, No. 76)

Local name: Bada, Bata; English name: Yellowtail Mullet; Habitat: River.

77. Rhinomugil corsula (Hamilton, 1822) (Plate 4, No. 77)

Local name: Bada, Bata; English name: Corsula Mullet; Habitat: Estuary.

\section{Order: 11. Scorpaeniformes}

\section{Familly: 32. Platycephalidae}

78. Platycephalus indicus (Linnaeus, 1758) (Plate 4, No. 78) Local name: Chota Byla, Mur Byla; English name: Bartail Flathead; Habitat: River and estuary.

\section{Order: 12. Synbranchiformes}

\section{Familly: 33. Mastacembelidae}

79. Mastacembelus armatus (Lacepède, 1800) (Plate 4, No. 79) Local name: Kata Baim; English name: Zig-zag Eel; Habitat: Wide freshwater; IUCN status: Endangered.

80. Macrognathus pancalus Hamilton, 1822 (Plate 4, No. 80)

Local name: Guci Baim; English name: Barred Spiny Eel; Habitat: Wide freshwater.

81. Macrognathus aculeatus (Bloch, 1786) (Plate 4, No. 81)

Local name: Tara Baim; English name: Lesser Spiny Eel; Habitat: Wide freshwater.

\section{Order: 13. Pleuronectiformes}

Familly: 34. Soleidae

82. Brachirus orientalis (Bloch \& Schneider, 1801) (Plate 4, No. 82)

Local name: Kukur Jib; English name: Oriental Sole; Habitat: River and estuary.

\section{Familly: 35. Cynoglossidae}

83. Cynoglossus cynoglossus (Hamilton, 1822) (Plate 4, No. 83)

Local name: Kukur Jib; English name: Bengal Tongue Sole; Habitat: River and estuary. 


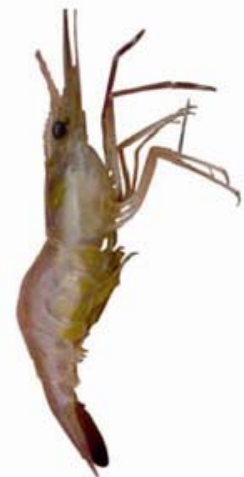

84. Macrobrachium villosimanus

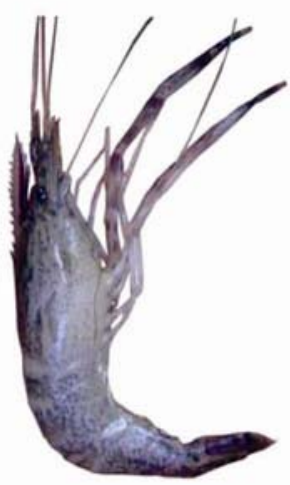

87. Macrobrachium dolichodactvlus

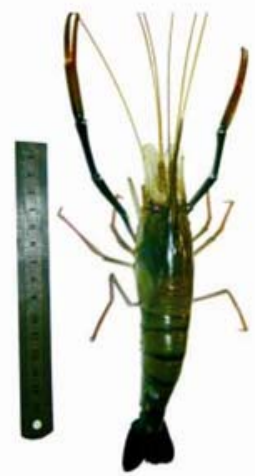

85. Macrobrachium rosenbergii

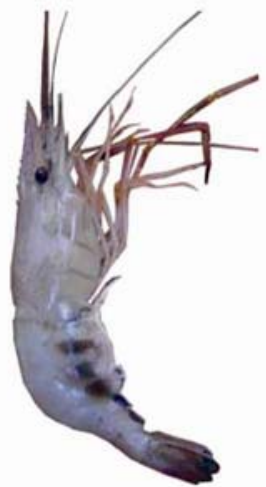

88. Macrobrachium davanus

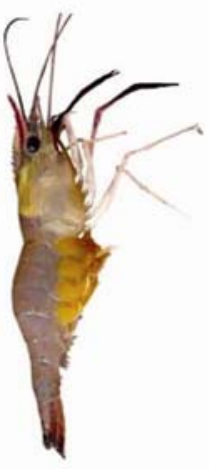

86. Macrobrachium malcolmsonii

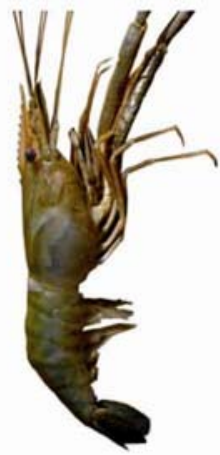

89. Macrobrachium rude

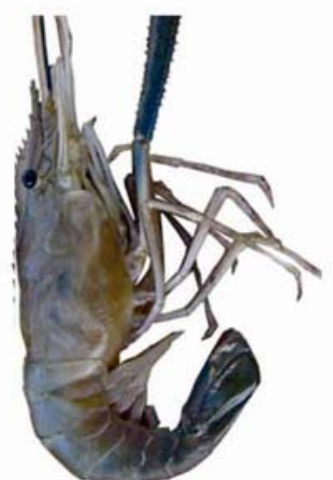

90. Macrobrachium birmanicum

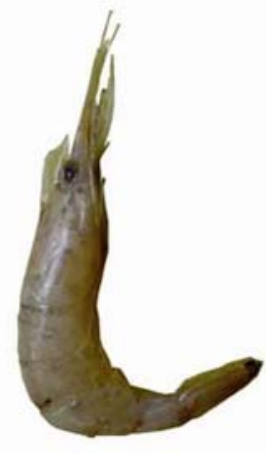

91. Macrobrachium mirabilis

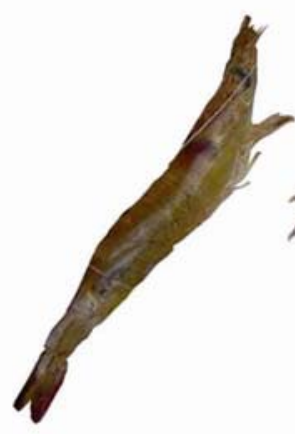

92. Metapenaeus monoceros

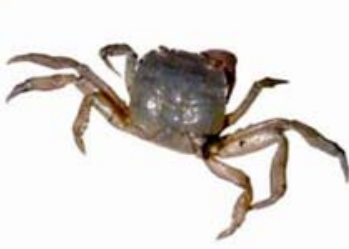

93. Potamon martensi

Plate 5. Shellfishes (Crustaceans) of the River Halda 


\section{Phylum: Arthropoda \\ Class: Crustacea \\ Order: Decapoda}

\section{Family: 1. Palaemonidae}

1. Macrobrachium villosimanus (Tiwari, 1947) (Plate 5, No. 84)

Local name: Dimua Icha, English name: Dimua River Prawn; Habitat: River.

2. Macrobrachium rosenbergii (de Man 1879) (Plate 5, No. 85)

Local name: Golda Chingri, English name: Giant River Prawn; Habitat: River and estuary.

3. Macrobrachium malcolmsonii (H.M.Edwards, 1844)(Plate 5, No.86)

Local name: Chotka Icha, Tora Icha, English name: Monsoon River Prawn; Habitat: River and estuary.

4. Macrobrachium dolichodactylus (Hilgendorf, 1878) (Plate 5, No. 87)

Local name: Ghoda Icha, English name: Ghoda River Prawn; Habitat: Freshwater.

5. Macrobrachium dayanus (Henderson, 1893) (Plate 5, No. 88)

Local name: Ghoda Icha, English name: Kaira River Prawn; Habitat: River.

6. Macrobrachium rude (Heller, 1862) (Plate 5, No. 89)

Local name: Paitta Icha, English name: Hairy River Prawn; Habitat: River, estuary and sea.

7. Macrobrachium birmanicum (Schenkel, 1902) (Plate 5, No. 90)

Local name: Ghoda Icha, English name: Birma River Prawn; Habitat: Freshwater.

8. Macrobrachium mirabilis (Kemp, 1917) (Plate 5, No. 91)

Local name: Lotia Icha, English name: Short-leg River Prawn; Habitat: River and estuary.

\section{Family: 2. Penaeidae}

9. Metapenaeus monoceros (Fabricius, 1798) (Plate 5, No. 92)

Local name: Harina Icha, Lolia Icha, English name: Brown Shrimp; Habitat: Sea and estuary.

\section{Family: 3. Portunidae}

10. Potamon martensi (Wood Mason, 1871) (Plate 5, No. 93)

Local name: Cimta Kakra, English name: Crab, Habitat: Freshwater.

Eighty-three finfish and ten shellfish (nine prawns and one crab) species were identified in this study. Rainboth (1978) studied the fishes from catch compositions of three habitats of the Halda and Ichamati area, [i) standing water 
habitat (includes floodplains of Halda and Ichamati), ii) upland river habitat (Ichamati River), and iii) Tidal stream habitat (Halda River)] and documented 78 species of finfish and 12 species of prawn where 48 finfish and 8 prawn species from the floodplains, 23 finfish and 5 prawn species from the Ichamati river, and 65 finfish and 12 prawn species from the Halda river. Rainboth (1978) again listed 99 species of finfish and 12 species of prawn from Halda and Ichamati area combined during winter season without mentioning catch composition or other details in the same working documents.

In the present study although finfish species (83) richness in the River Halda is higher with more 18 species in comparison to 65 species of Rainboth (1978) but 23 species of Rainboth (1978) was absent during present investigation. Among the absent 23 finfish species, three are coastal (Collia sp, Leiognathus eqqulus, Herpaodon nehereus) and four are floodplains (Esomus danricus, Dermogenys pusilla, Chela cachius and Oreichthys cosuatis), which might be the occasional invader to Halda river. Moreover, among the 23 absent species, nine were wide freshwater (Oryzias malaonostigma, Denio rerio, Rasbora daniconius, Puntius chola, Tetraodon cutcutia, Mystus bleekeri, Badis badis, Puntius guganio, and Colisa lalia), which seemed to be recorded from the Halda adjacent flood plains. Among other absent fishes, two were up riverine (Barilus barna and Gagata gagata), four estuarine (Pama pama, Awaous stamineus, Eleotris fusca, and Synuaptura pan) and one (Taeniodes gracilisis) is questionable as there is no other records. Due to local short distance migratory nature of (tropical) freshwater fish species, a large number of blackfishes (limnophilic freshwater species that migrates to river) were somewhat frequently found in river and whitefishes (rheophilic species that migrate to the floodplain) in flood plain. Thus flood plain fishes that recorded earlier by Rainboth (1978) from the river Halda can not be concluded as disappeared but during the present study was not found in the catch composition of Halda River. Further investigation on the fish fauna of floodplain, upland river, tributaries and coastal water could be able to conclude whether these species are disappeared or not.

Of the 83 finfish species recorded in this study, three belongs to the critically endangered, nine endangered and eight vulnerable categories according to IUCN (2000). Four finfishes (Moringua macrocephalus, Oryzias dancena, Ophieleotris aporos and Opistognathus nigromarginatus) are the new records from inland waters of Bangladesh. Another species Labeo ariza was also available in lesser extent in the River Halda, which was not reported by most of the earlier authors except Ahmed et al. (2007) and Haque et al. (2007) from the some rivers of Netrokona. 
Some exotic fishes which are found in the river Halda were also reported from other inland open waters of Bangladesh both from lotic and lentic habitats viz. from BKSB beel of Khulna by Rahman et al. (1999), from Kaptai lake by Ahmed et al. (2001), from Maljhee-Kangsa floodplain of Sherpur district by Ahmed et al. (2005), from Dopi beel in Joanshahi Haor of Kishorgonj by Azhar et al. (2007), from River Titas by Ahmed and Akhter (2008), from Titas floodplain by Ahmed (2008), and from Mahananda river by Mohsin and Haque (2009).

The finfish (83 species) and shellfish (9 prawn) species of Halda river were higher than those of Pagla river (75 finfish) (Zafar et al. 2007), Mahananda river (56 finfish) (Mohsin and Haque 2009), Irrawady river, Myanmar (79 finfish), Narmada, India (77 finfish), Sepik (New Guinea) (De Silva et al. 2007). But the number of fish species in the river Naaf (98 finfish and 23 prawns - Hossain et al. 2007) and Padma (110 finfish - Islam and Hossain 1983) were higher than those of the river Halda. It might be due to the directly linkage with the sea in case of river Naaf, where marine species were included and in case of Padma due to vast area of the river. However, while comparing earlier records of Rainboth (1978) with the present one, species richness in the Halda River was found to be 120 (106 finfish, 14 prawns), which is comparable with larger river systems of Asia.

Tidal River Halda is well known as a unique spawning ground of major carps in Bangladesh from where fertilized eggs of major carps are collected and hatched in the mud made scoop on the river bank thus producing considerable number of fries for the country's aquaculture. The River is also well known for producing brood of freshwater giant prawn and other fishery. The river habitat and its living beings are under threat due to unscientific human interventions like loop cutting, blocking natural flow of water of big tributaries by making sluice gates, embankment, pollution from paper mill (Chittagong Asian paper mill) and power plant (Picking power plant), rubber dam over the river at upstream, as well natural causes like siltation, erosion, climate change (irregular and untimely rainfall, drought, temperature increase). Since 2007 the Government has declared Halda River as fish sanctuary to protect the spawning ground of major carps and its fisheries resources. To conserve and protect the resources of this important river, declared regulations should be implemented strictly and properly and illegal fishing should be stopped by the law enforcement agencies and by creating public awareness. Present records of 93 ichthyofauna (83 finfishes and 10 shellfish species) will help the future researchers, planners and policy makers as no published account of ichthyofauna of Halda river is available after or before major interventions (12 sluice gates over 12 major tributaries of Halda River, blocking the migration and 
local movement of fish) during 1975-'76 and 1982-'83 by Bangladesh Water Development Board for irrigation and flood control.

Acknowledgements: The authors are grateful to the Ministry of Education, Government of the Peoples' Republic of Bangladesh for providing support under the Grants for Advanced Research in Science. The second author is also grateful to the University Grants Commission of Bangladesh for partial support. The Department of Zoology, University of Chittagong has provided technical laboratory support.

\section{LITERATURE CITED}

AHMED, B. and HASAN, S. 1981. A checklist of the fishes of the Karnaphuli Reservoir. Bangladesh J. Zool. 9(1): 37-40.

AHMED, K.K.U., HAMBREY, J.B. and RAHMAN, S. 2001. Trends in interannual yield variation of reservoir fisheries in Bangladesh, with special reference to Indian Major carp. Lakes and reservoir: Research and management. 6: 85-94

AHMED, K.K.U., AHAMED, S.U., HASAN, K.R. and MUSTAFA, M.G. 2007. Option for formulating community based fish sanctuary and its management in beel ecosystem in Brahmanbaria. Bangladesh J. Fish. (Special Issue, 2007) 30: 1-10.

AHMED, M.S, and AKTHER, H. 2008. Brush and vegetation park fishery in the River Titas, Brahmanbaria, Bangladesh. South Pacific Studies, 29(1):63-71.

AHMED, M.S. 2008. Assessment of fishing practice on the exploitation of the Titas flood plain in Brahmanbaria. Bangladesh. Turkish J. Fish. Aquat Sci. 8(2):329-334.

AHMED, N. 1948. Methods of collection and hatching of carp ova in Chittagong with some suggestion for their improvement. J. Bombey Nat. Hist. Soc. 47(4): 593-602.

AHMED, N. 1953. Fish fauna of East Pakistan. Pak. J. Sci, Lahore 5(1):18-24.

AHMED, N. 1955. Certain observation on the spawaning of major carps in the River Halda of Chittagong (East Pakistan). J. Zool. Soc. India. 1: 101-103

AHMED, N. 1957. Prawn and prawn fishery of East Pakistan. Directorate of Fisheries, Govt. E. Pak. Publ. No. 11-43: 1-43 pp.

AHMED, N., RAHMAN, M.M. and RAHMAN, M.M. 2005. Fish catch assessment of Maljehee-Kangsa floodplain in Bangladesh. Pakistan Journal of Biological sciences 8(3) 396-400.

ALI, L. and MORRIS, E.L. 1977. Results of the economic and catch assessment program (First survey) in the Chandpur, Muhuri, Halda and Ichamati project areas. Development of fisheries in the Chandpur, Muhuri and Karnafuli (Halda and Ichamati) irrigation and flood control project, working document No.4, Directorate of Fisheries Bangladesh with Snell environmental group, Inc. Lansing, Michigan, U.S.A.

ALI, L. and MORRIS, E.L. 1978. Results of the economic and catch assessment program (Second survey) in the Chandpur, Muhuri, Halda and Ichamati project areas. Development of fisheries in the Chandpur, Muhuri and Karnafuli (Halda and Ichamati) irrigation and flood control project, working document No.7, Directorate of Fisheries Bangladesh with Snell environmental group, Inc. Lansing, Michigan, U.S.A.

ARSHAD-UL-ALAM, M. 2013. Fishing intensity of the Halda River, Chittagong, Bangladesh. Int. J. Sci. Inn. Tech. Sec. A. 2(5): 10-16. 
AZADI, M.A. 2004. Management of spawn fishery of major carps (Labeo rohita, Catla catla and Cirrhinus cirhosus) in the Halda River, Chittagong, Bangladesh. Bangladesh J. Fish. (Special Issue) 27: 50-51.

AZADI, M.A. and ARSHAD-UL- ALAM, M. 2011. Diversity of Finfish and Shellfish of the River Halda with Notes on their Conservation. P. 92-102. In Eivin Røskaft and David J. Chivers (Eds.), Proc. International Conf. on Biodiversity-Present State, Problems and Prospects of its Conservation. Norwegian Center for International Cooperation in Education (SIU), NO 5809, Bergen, Norway. Tapir Academic Press, Trondheim, Norway.

AZHAR, S.A., DEWAN, S., WAHAB, M.A., HABIB, M.A.B., and MUSTAFA, M.G. 2007. Impacts of fish sanctuaries on production and biodiversity of fish and prawn in Dopi beel, Joanshahi haor, Kishoregonj. Bangladesh J. Fish (Special issue, 2007) 30: 23-36.

BERG, L.S. 1940. Classification of fishes, both recent and fossil. Trav. Inst. Zool. Acad. Sci. U.S.S.R., (Russian English texts, Also reprint, Ann Arbor, Michigan, 1974) 517. pp.

BHUIYAN, A.L. 1964. Fishes of Dacca. Asiatic Soc. Pakistan Publ., Dacca. No. 13, 148. pp.

CHONG, K.C. 1979. An economic appraisal of the fisheries in the Chandpur, Muhuri, Halda and Ichamati project areas. Development of fisheries in the Chandpur, Muhuri and Karnafuli (Halda and Ichamati) irrigation and flood control project, working document no.13, 19 pp. Directorate of Fisheries Bangladesh with Snell environmental group, Inc. Lansing, Michigan, U.S.A.

DAY, F. 1889. The fauna of British India Including Ceylon and Burma: Fishes Vol. I, pp. 548, and Vol. II, pp. 509. Toylor and Francis, London.

DE SILVA, S.S., ABERY, N.W. and NGUYEN, T.T.T. 2007. Endemic freshwater finfish of Asia: distribution and conservation status. Diversty and distribution. 13: 172-184.

DoF. 2013. Fish fortnight issue, Department of Fisheries, Govt. People's Rep. of Bangladesh.

DOHA, S. 1973. Fishes of the district of Mymensingh and Tangail. Banladesh J. Zool. 1(1): 1-10.

FINCHAM, A.A. and WICKINS, J.F. 1976. Identification of commercial prawns and shrimps. British Mus. Publ. $779: 7$ pp.

GEORGE, M. 1969. Prawn fisheries of India, II. Systematics-Taxonomic consideration. Bull. Cent. Mar. Fish. Res. Inst.: 5-48.

HAFIZUDDIN, A.K.M., MAHMOOD, N., and AZADI, M.A. 1989. An addition to the Ichthyofauna of Kaptai Lake. Bangladesh J. Zool. 17(1): 29-33.

HAQUE, M., AHMED, M., and MUSTAFA M.G. 2007. Preference of fishes to different types of Katha materials used in sanctuaries in three rivers of Netrokona. Bangladesh J. Fish (Special issue, 2007) 30:153-163.

HAROON, A.K.Y., KHAN, A.A., HALDER, G.C., and RAINBOTH, W.J. 1989. A checklist of the fish and prawn fauna of the Muhuri irrigation project and surrounding region. Bangladesh J. Zool. 17(1): 19-28.

HOSSAIN, M.S., DAS N.G. and CHOWDHURY M.S.N 2007. Fisheries management of the Naaf River. Coastal and Ocean Research Group of Bangladesh (CORG), Institute of Marine Science and Fisheries, University of Chittagong, $257 \mathrm{pp}$.

HOSSAIN, M.S. 2013. An illustrated guide to Fishes of Noakhali. Tetra-C, Chittagong, Bangladesh, $276 \mathrm{pp},+32$ colour plates.

HOWLADER, M.S. 1976. A taxonomic account of penaeid shrimp of Bangladesh waters. Bangladesh J. Sci. \& Ind. Res. 11(1-4): 122- 138.

ISLAM, M.S. and HOSSAIN , M.A. 1983. An account of the fisheries of the Padma near Rajshahi. Raj. Fish Bull. 1(2):1-3.

IUCN BANGLADESH 2000. Red list of threatened animals of Bangladesh. IUCN- The world Conservation Union. Xii +54 pp. 
JAYARAM, K.C. 1981. The freshwater fishes of India, Pakistan, Bangladesh, Burma, and Sri LankaA Handbook. Zoological Survey of India, Calcutta, XXII +475 pp. 13 Pls.

KHAN, S. and AZADI, M.A. 2006. Studies and management strategies to revive and sustain the fisheries in the River Halda, A research Project of MoFL and WorldFish Centre.

LAGLER, K.F. 1962. Ichthyology. John Wiley and Sons (1962), Inc., New York- London. 545. pp

MOHSIN A.B.M., and HAQUE, E., 2009. Diversity of fishes of Mahananda river at Chapai Nawabgonj district. Res. J. Biol. Sci. 4(7) 828-831, 2009.

MUNRO, I.S.R. 1955. Marine and freshwater fishes of Ceylon, Department of External Affairs, Canberra. 351. pp.

PATRA, R.W.R. and AZADI, M.A. 1984. Collection and hatching of fertilized eggs of major carps. Chittagong Univ. Stud. Part II Sc. 8(1):45-49.

PATRA, R.W.R. and AZADI M.A. 1985a. Limnology of Halda River. The Journal of NOAMI 2(2): 31-38.

PATRA, R.W.R. and AZADI M.A. 1985b. Hydrological condition influencing the spawning of major carps in the Halda River Chittagong, Bangladesh. Bangladesh J. Zool. 13(1):63- 72.

PATRA, R.W.R. and AZADI M.A. 1987. Ecological studies on the planktonic organisms of the Halda River Chittagong, Bangladesh. Bangladesh J. Zool. 15(2): 109-123.

RAHMAN, S., MAZID M.A., KAMAL M., HOSSAIN M.A. and HOSSAIN M.S. 1999. Study on fishing gears, species selectivity towards gears and catch composition of BSKB beel, Khulna, Bangladesh. Bangladesh J. Fish. Res. 3(1):25-32.

RAHMAN, A.K.A. 1975. A check-list of the freshwater bony fishes of Bangladesh. Bulletin No. 1, Freshwater Fisheries Research Station, Chandpur, Directorate of Fisheries, Bangladesh. Bangladesh Government Press, Dacca, pp. 1-18.

RAHMAN, A.K.A. 1989. Fresh water Fishes of Bangladesh. Zool. Soc. Bangladesh, Dept. of Zoology, University of Dhaka. 364 pp.

RAHMAN, A.K.A. 2005. Freshwater fishes of Bangladesh. $2^{\text {nd }}$ ed., Zool. Soc. Bangladesh, Dhaka, Bangladesh. 394 pp.

RAHMAN, S.M. 2013. The Halda: A River with Unique Characteristics. Pages 61-64. In DoF 2013 National Fish Week 2013 Compendium (In Bengali). Department of Fisheries, Ministry of Fisheries and Livestock, Bangladesh. 144 p.

RAINBOTH, W.J. 1978. The fishes and prawns of Halda and Ichamati units of the Karnafuli flood control and irrigation project, with reference to potential impacts of the development scheme. Development of fisheries in the Chandpur, Muhuri and Karnafuli (Halda and Ichamati) irrigation and flood control project, working document no.18.Directorate of Fisheries Bangladesh with Snell environmental group, Inc. Lansing, Michigan, U.S.A.

SHAFI, M. and QUDDUS, M.M.A. 1982. Bangladesher Matsho Sampad (Fisheries of Bangladesh, in Bengali). Bangla Academy, Dacca, Bangladesh. 444 pp.

TALWAR, P.K. and HINGRAN A.G. 1991. Inland Fishes of India and adjacent countries. CRC Press, Vol. I \& II. 1158 pp.

ZAFAR, M., AMIN, S.M.N. and IQBAL, M.J. 2007. Biodiversity of fisheries organism in the Pagla river of Bangladesh. Bangladesh J. Fish. (special issue, 2007) 30:165-175. 